\title{
O Impacto da Incontinência Urinária na Autoestima e Autoimagem de Pacientes Diabéticos
}

\author{
The Impact of Urinary Incontinence on the Self-esteem and \\ Self-image of Patients with Diabetes
}

\author{
El Impacto de la Incontinencia Urinaria en la Autoestima \\ y Autoimagen de Pacientes Diabéticos
}

\author{
Geraldo Magela Salomé1, Thaís Fátima de Oliveira², Wesley Alexandre Pereira²
}

\begin{abstract}
RESUMO
Objetivo: Avaliar autoestima e autoimagem em 20 pacientes diabéticos com incontinência urinária, atendidos em um Centro de Educação em Diabetes no Brasil. Métodos: Os critérios de inclusão foram: incontinência urinária clinicamente diagnosticada; idade $\geq 18$ anos; condições físicas e mentais para responder aos questionários; e ter adquirido incontinência urinária após o diagnóstico de diabetes. O critério de exclusão foi incontinência fecal. Três instrumentos foram utilizados para a coleta de dados: um questionário sobre dados sociodemográficos e características das perdas urinárias, a Escala de Investimento Corporal e a Escala de Autoestima Rosenberg da Escola Paulista de Medicina da Universidade Federal de São Paulo. O teste do $\chi^{2}$ foi utilizado para avaliar a distribuição proporcional das variáveis sociodemográficas e de características da incontinência urinária, ou seja, se havia diferença na proporção de pacientes entre as categorias de uma variável. Também foram utilizados o teste de Kruskal-Wallis e o coeficiente de correlação de Spearman. O nível de significância foi estabelecido em $5 \%(p<0,05)$. Resultados e Conclusão: Pacientes com incontinência urinária apresentaram baixa autoestima e baixos escores nos domínios 'imagem corporal' e 'toque pessoal da Escala de Investimento Corporal', mostrando sentimentos negativos em relação ao próprio corpo.
\end{abstract}

DESCRITORES: Estomaterapia. Incontinência urinária. Autoimagem. Imagem corporal.

\begin{abstract}
Objective: To evaluate the self-esteem and self-image in 20 patients with diabetes and urinary incontinence who were cared at a Diabetes Education Center in Brazil. Methods: The inclusion criteria were: clinical diagnosis of urinary incontinence; age $\geq 18$ years; physical and mental conditions to answer the questionnaires; and onset of urinary incontinence after the diagnosis of diabetes. Exclusion criterion was fecal incontinence. Three instruments were used for data collection: a questionnaire on sociodemographic data and characteristics of urinary incontinence, the Body Investment Scale and the Rosenberg Self-Esteem Scale of Escola Paulista de Medicina from Universidade Federal de São Paulo. The $\chi^{2}$ test was used to evaluate the proportional distribution of sociodemographic variables and characteristics of urinary incontinence, or in other words, if there was a difference in the proportion of patients across categories of a variable. The Kruskal-Wallis test and Spearman correlation coefficient were also used. The level of significance was set at $5 \%(p<0.05)$. Results and Conclusion: Patients with urinary incontinence had low self-esteem and low scores in the 'body image' and 'personal touch' domains of the Body Investment Scale, showing negative feelings about their own bodies.
\end{abstract}

DESCRIPTORS: Stomatherapy. Urinary incontinence. Self concept. Body image.

\footnotetext{
${ }^{1}$ Universidade do VAle do Sapucaí (UNIVÁS) - Pouso Alegre (MG), Brasil. Endereço para correspondência: Avenida Francisco de Paula Quintanilha Ribeiro, 280, apto. 134 - Jabaquara - CEP: 04330-020 - São Paulo (SP), Brasil - E-mail: salomereiki@yahoo.com.br

²Departamento de Enfermagem da UNIVÁS - Pouso Alegre (MG), Brasil.

Artigo recebido em: 18/05/2015 - Aceito para publicação em: 01/12/2015
} 


\section{RESUMEN}

Objetivo: Evaluar la autoestima y la autoimagen en 20 pacientes diabéticos con incontinencia urinaria, que habían sido tratados en un Centro de Educación en Diabetes en Brasil. Métodos: Los criterios de inclusión fueron: incontinencia urinaria diagnosticada clínicamente; edad $\geq 18$ años; condiciones físicas y mentales para responder a los cuestionarios; e inicio de la incontinencia urinaria después del diagnóstico de diabetes. El criterio de exclusión fue la incontinencia fecal. Tres instrumentos fueron aplicados para la recolección de datos: un cuestionario sobre los datos sociodemográficos y las características de las pérdidas de orina, la Escala de Investimento Corporal y la Escala de Autoestima Rosenberg de la Escola Paulista de Medicina de la Universidade Federal de São Paulo. Se utilizó la prueba del $\chi^{2}$ para evaluar la distribución proporcional de las variables sociodemográficas y de las características de incontinencia urinaria, es decir, si había una diferencia en la proporción de pacientes entre las categorías de una variable. Se utilizaron también la prueba de Kruskal-Wallis y el coeficiente de correlación de Spearman. El nivel de significancia se fijó en los 5\% (p<0,05). Resultados y Conclusiones: Los pacientes con incontinencia urinaria tuvieron una baja autoestima y puntuaciones bajas en los dominios de 'la imagen corporal' y 'del toque personal de la Escala de Investimento Corporal', mostrando sentimientos negativos acerca de su propio cuerpo.

DESCRIPTORES: Estomaterapia. Incontinencia urinaria. Autoimagen. Imagen corporal.

\section{INTRODUÇÃO}

A continência pode ser considerada uma condição essencial para o indivíduo de forma individual, em ambiente familiar e na sociedade. Relatos de pessoas que têm pouco ou nenhum controle esfincteriano mostram que esse problema resulta em sentimentos de vergonha e situações vexatórias capazes de interferir nos hábitos de vida e influenciar no convívio social. Por ser considerada para alguns como parte do envelhecimento, as queixas são sub-relatadas aos profissionais de saúde, os quais também as investigam de forma reduzida, talvez por desconhecimento de sua epidemiologia ${ }^{1-3}$.

Vários estudos apontam o impacto negativo sobre a qualidade de vida das pessoas acometidas por essas perdas. Apesar das incontinências urinária e fecal não causarem risco vital à saúde, podem ocasionar isolamento social, diminuição da autoestima e situações constrangedoras em público $^{1-4}$. Pesquisas acerca da incontinência apresentam, em maior número, perdas urinárias e fecais para mulheres $\geq 60$ anos e os meios de tratamento, em função da prevalência ser maior nesse grupo ${ }^{5}$. Para a incontinência combinada, os estudos são escassos em nível internacional. No Brasil, encontrou-se somente uma publicação até o final da realização deste trabalho ${ }^{6}$.

Quanto ao diabetes mellitus, um estudo realizado em 2001, no Ambulatório de Diabetes da Clínica Médica da Faculdade de Medicina de Ribeirão Preto da Universidade de São Paulo (FMRP-USP), revelou uma alta prevalência de sintomas digestivos nessa população específica, com sintomas de incontinência fecal (4,5\%), constipação $(16,9 \%)$ e diarreia
$(7,2 \%)$ em uma amostra de 153 pessoas $^{6}$. Em uma investigação sobre a caracterização de idosos diabéticos atendidos na atenção secundária em 2007, a incontinência fecal foi encontrada a partir do quinto ano de diagnóstico do diabetes, em 2,4\% de uma amostra de 113 pessoas $^{7}$. Para a incontinência urinária, em uma amostra de 910 mulheres, 237 eram diabéticas. Essa patologia estava associada a aumento de risco para tal enfermidade, uma vez que a incontinência urinária de esforço e a mista foram mais prevalentes em mulheres diabéticas do que em não diabéticas ${ }^{8}$.

Foi realizada uma revisão de literatura internacional e nacional, em que se detectou uma escassez de estudos que avaliam a autoestima e a autoimagem nos indivíduos diabéticos com incontinência urinária. Portanto, o objetivo deste estudo foi avaliar a autoestima e a autoimagem em indivíduos diabéticos com incontinência urinária.

\section{MÉTODO}

Trata-se de um estudo descritivo, analítico e transversal. A pesquisa foi desenvolvida em um Centro Municipal de Educação em Diabetes (CEMED), localizado em Pouso Alegre, Brasil. Os dados foram coletados entre dezembro de 2012 e maio de 2013, após aprovação pelo Comitê de Ética em Pesquisa da Universidade do Vale do Sapucaí, sob parecer 634.020. A amostra foi selecionada de forma não probabilística, por conveniência. Vinte pacientes diabéticos foram incluídos no estudo, após assinarem o termo de consentimento livre e esclarecido. 
Os dados foram coletados pelos próprios pesquisadores, em uma sala previamente preparada, garantindo o conforto e a tranquilidade do paciente para a emissão das respostas.

Os critérios de inclusão foram: incontinência urinária clinicamente diagnosticada; idade $\geq 18$ anos; condições físicas e mentais para responder aos questionários e ter adquirido incontinência urinária após o diagnóstico de diabetes. $\mathrm{O}$ critério de não inclusão foi incontinência fecal.

As informações foram coletadas por meio de três instrumentos. O primeiro refere-se aos dados sociodemográficos (sexo, etnia, escolaridade, situação conjugal, ocupação e renda familiar) e clínicos (cirurgia uroginecológica prévia, disúria, infecção urinária prévia, alterações genitais e medicamentos) da amostra Além disso, incluiu algumas características de perdas urinárias, tais como: período e condições das perdas; chegar a tempo no banheiro; acordar durante a noite para urinar; perdas urinárias durante a relação sexual; frequência e quantidade de perdas urinárias; utilização e frequência das trocas de recursos de contenção e de aspectos subjetivos; mudanças em hábitos de vida diária e relacionamento social ${ }^{9}$.

O segundo recurso foi utilizado para avaliar a autoimagem e consiste na versão brasileira da Escala de Investimento Corporal (Body Investment Scale) composta por 20 itens, divididos em três fatores: imagem, cuidado e toque corporais. As respostas estão dispostas numa escala Likert de cinco pontos, que varia de "discordo totalmente" (um ponto) a "concordo totalmente" (cinco pontos). Para obter o escore final da escala, devem-se reverter aqueles dos itens 2, 5, 9, 11,13 e 17 e somá-los. O valor total varia entre 20 e 100. Os escores nos domínios "imagem corporal" e "toque corporal" variam entre 6 e 30 , e em "cuidado corporal", entre 8 e 40. Quanto maior a pontuação, maior é o sentimento positivo em relação ao corpo ${ }^{10}$.

O terceiro instrumento consistiu na Escala de Autoestima Rosenberg da Escola Paulista de Medicina da Universidade Federal de São Paulo (UNIFESP-EPM). A versão brasileira é um instrumento específico para a medida de autoestima, composto por dez questões fechadas, com as seguintes opções de respostas: concordo plenamente, concordo, discordo e discordo plenamente. $O$ escore total varia de 0 a 30, sendo que zero refere-se à melhor autoestima e 30 , a pior $^{11}$.

Os dados coletados foram organizados em um banco de dados (Microsoft Excel) e analisados no programa Statistical Package for the Social Sciences (SPSS) versão 15.0. O teste do $\chi^{2}$ foi utilizado para analisar a distribuição proporcional das variáveis sociodemográficas e das características da incontinência urinária, ou seja, se havia diferença na proporção de pacientes entre as categorias de uma variável. Também foram utilizados o teste de Kruskal-Wallis e o coeficiente de correlação de Spearman. O nível de significância foi estabelecido em $5 \%(\mathrm{p}<0,05)$.

\section{RESULTADOS}

A maioria dos participantes era do sexo feminino e com faixa etária acima de 60 anos; 8 (40\%) eram casados e 17 (85\%) eram fumantes (Tabela 1 ).

Com relação aos dados clínicos, a maioria dos participantes da pesquisa possuía alterações no órgão genital; nunca havia realizado cirurgia ginecológica ou retal e não sentia dor ou ardências para urinar. Uma grande parte dos pacientes nunca foi ao médico para tratar a incontinência urinária; acorda à noite para urinar; tem perda de urina ao tossir e sente sensação de estar úmido, porém não urina durante a relação sexual (Tabela 2).

Ao analisar a Tabela 3, observa-se que a média do escore na Escala de Investimento Corporal foi de 82,80 e na Escala de Autoestima Rosenberg/UNIFESP-EPM, 24,95 . Os totais médios nos domínios 'imagem corporal' e 'toque corporal'da Escala de Investimento Corporal foram baixos, caracterizando que os pacientes deste estudo apresentaram alterações na imagem corporal.

\section{DISCUSSÃO}

O aumento da expectativa de vida, a industrialização e os efeitos da urbanização fizeram com que a população brasileira ficasse mais exposta a problemas de saúde, dentre os quais, destacam-se câncer, diabetes mellitus, feridas, incontinências urinária e fecal, hipertensão arterial, acidente vascular cerebral, mal de Parkinson e outras doenças crônicas, nas quais, muitas vezes, os portadores dessas moléstias apresentam incontinência urinária. Ser idoso, diabético e ter de conviver com esta enfermidade faz a pessoa sentir-se impotente, frágil, com medo e com alteração na qualidade do sono. Tais sentimentos têm como consequência mudanças na qualidade de vida, na autoimagem e na autoestima ${ }^{12-19}$.

No presente trabalho relacionado à caracterização sociodemográfica dos indivíduos, verificou-se que a maioria da amostra foi constituída por pessoas idosas, com média de 
Tabela 1. Características demográficas dos pacientes com incontinência urinária.

\begin{tabular}{|c|c|c|c|c|c|c|}
\hline Variáveis & $\mathrm{n}$ & Média & Mediana & DP & Mínima & Máxima \\
\hline Idade & 20 & 64,30 & 62,00 & 9,576 & 48 & 86 \\
\hline & $\mathrm{n}$ & $\%$ & $\%$ válido & \multicolumn{2}{|c|}{$\%$ acumulado } & Valor $\mathrm{p}$ \\
\hline \multicolumn{7}{|c|}{ 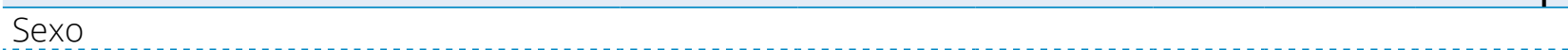 } \\
\hline Masculino & 3 & 15,0 & 15,0 & & 15,0 & \multirow{2}{*}{$0,027 *$} \\
\hline Feminino & 17 & 85,0 & 85,0 & & 100 & \\
\hline Total & 20 & 100 & 100 & & & \\
\hline \multicolumn{7}{|l|}{ Estado civil } \\
\hline Casado & 8 & 40,0 & 40,0 & & 40,0 & \multirow{5}{*}{0,237} \\
\hline Separado & 3 & 15,0 & 15,0 & & 55,0 & \\
\hline Solteiro & 3 & 15,0 & 15,0 & & 70,0 & \\
\hline Viúvo & 6 & 30,0 & 30,0 & & 100 & \\
\hline Total & 20 & 100 & 100 & & & \\
\hline \multicolumn{7}{|l|}{ Fumante } \\
\hline $\mathrm{sim}$ & 17 & 85,0 & 85,0 & & 35,0 & \multirow{2}{*}{0,027 * } \\
\hline Não & 3 & 15,0 & 15,0 & & 100 & \\
\hline Total & 20 & 100 & 100 & & & \\
\hline \multicolumn{7}{|l|}{ Etnia } \\
\hline Negra & 1 & 5,0 & 5,0 & & 5,0 & \multirow{3}{*}{$0,043 *$} \\
\hline Amarela & 5 & 25,0 & 25,0 & & 30,0 & \\
\hline Branca & 14 & 70,0 & 70,0 & & 100 & \\
\hline Total & 20 & 100 & 100 & & & \\
\hline \multicolumn{7}{|l|}{ Escolaridade } \\
\hline Alfabeto & 1 & 5,0 & 5,0 & & 5,0 & \multirow{5}{*}{0,031 * } \\
\hline Ensino médio incompleto & 2 & 10,0 & 10,0 & & 15,0 & \\
\hline Fundamental incompleto & 16 & 80,0 & 80,0 & & 35,0 & \\
\hline Superior completo & 1 & 5,0 & 5,0 & & 100 & \\
\hline Total & 20 & 100 & 100 & & & \\
\hline \multicolumn{7}{|l|}{ Ocupação } \\
\hline Aposentado & 10 & 50,0 & 50,0 & & 50,0 & \multirow{5}{*}{0,067} \\
\hline Do lar & 7 & 35,0 & 35,0 & & 35,0 & \\
\hline Auxiliar de limpeza & 2 & 10,0 & 10,0 & & 5,0 & \\
\hline Pintor & 1 & 5,0 & 5,0 & & 100 & \\
\hline Total & 20 & 100 & 100 & & & \\
\hline \multicolumn{7}{|l|}{ Renda familiar } \\
\hline s3 salários-mínimos & 19 & 95,0 & 95,0 & & 95,0 & \multirow{2}{*}{$0,007 *$} \\
\hline 4 a 6 salários-mínimos & 1 & 5,0 & 5,0 & & 100 & \\
\hline Total & 20 & 100 & 100 & & & \\
\hline \multicolumn{7}{|l|}{ Possuem filhos } \\
\hline $\mathrm{Sim}$ & 14 & 70,0 & 70,0 & & 70,0 & \multirow[t]{3}{*}{$0,021 *$} \\
\hline Não & 6 & 30,0 & 30,0 & & 100 & \\
\hline Total & 20 & 100 & 100 & & & \\
\hline \multicolumn{7}{|l|}{ Medicamentos em uso } \\
\hline Diurético/antidiarreico & 1 & 5,0 & 5,0 & & 5,0 & \multirow{9}{*}{0,431} \\
\hline Diurético & 6 & 30,0 & 30,0 & & 35,0 & \\
\hline Laxante/diurético hipotensor & 1 & 5,0 & 5,0 & & 40,0 & \\
\hline Antidiarreico & 1 & 5,0 & 5,0 & & 45,0 & \\
\hline Anti-histamínico/hipotensor & 1 & 5,0 & 5,0 & & 50,0 & \\
\hline Antidepressivo & 1 & 5,0 & 50,0 & & 5,0 & \\
\hline Hipoglicemiante & 4 & 20,0 & 20,0 & & 75,0 & \\
\hline Hipotensor & 5 & 25,0 & 25,0 & & 100 & \\
\hline Total & 20 & 100 & 100 & & & \\
\hline
\end{tabular}

*significância estatística - teste do $\chi^{2}(p \leq 0,05)$; `salário-mínimo = R\$788,00; DP: desvio padrão. 
Tabela 2. Características clínicas relacionadas à incontinência urinária.

\begin{tabular}{|c|c|c|c|c|c|}
\hline Variáveis & $\mathrm{n}$ & $\%$ & $\%$ válido & $\%$ acumulado & Valor $p$ \\
\hline \multicolumn{6}{|l|}{ Alteração no órgão genital feminino } \\
\hline Não possuem alterações & 16 & 80,0 & 80,0 & 80,0 & \multirow{2}{*}{0,041 * } \\
\hline Possuem fissura anal/hemorroida & 4 & 20,0 & 20,0 & 100 & \\
\hline Total & 20 & 100 & 100 & & \\
\hline \multicolumn{6}{|l|}{ Alteração no órgão genital masculino } \\
\hline Não possuem alterações & 19 & 95,0 & 95,0 & 95,0 & \multirow{2}{*}{$0,039 *$} \\
\hline Possuem trauma/ferimento & 1 & 5,0 & 5,0 & 100 & \\
\hline Total & 20 & 100 & 100 & & \\
\hline \multicolumn{6}{|l|}{ Cirurgia ginecológica ou retal prévia } \\
\hline Nunca realizou & 13 & 65,0 & 65,0 & 65,0 & \multirow{4}{*}{0,062} \\
\hline Esterectomia & 2 & 10,0 & 10,0 & 75,0 & \\
\hline Períneo & 2 & 10,0 & 10,0 & 85,0 & \\
\hline Salpingotripsia & 3 & 15,0 & 15,0 & 100 & \\
\hline Total & 20 & 100 & 100 & & \\
\hline \multicolumn{6}{|l|}{ Dor, ardência ou dificuldade para urinar } \\
\hline Sim & 8 & 40,0 & 40,0 & 40,0 & \multirow{2}{*}{0,49} \\
\hline Não & 12 & 60,0 & 60,0 & 100 & \\
\hline Total & 20 & 100 & 100 & & \\
\hline \multicolumn{6}{|l|}{ Já teve infecções urinárias } \\
\hline Sim & 5 & 25,0 & 25,0 & 25,0 & \multirow{2}{*}{$0,012 *$} \\
\hline Não & 15 & 75,0 & 75,0 & 100 & \\
\hline Total & 20 & 100 & 100 & & \\
\hline \multicolumn{6}{|l|}{ Visita ao médico por perda urinária } \\
\hline Nenhuma vez & 15 & 75,0 & 75,0 & 75,0 & \multirow{4}{*}{$0,037 *$} \\
\hline 1 a 2 vezes & 2 & 10,0 & 10,0 & 85,0 & \\
\hline 3 a 5 vezes & 2 & 10,0 & 10,0 & 95,0 & \\
\hline$>10$ vezes & 1 & 5,0 & 5,0 & 100 & \\
\hline Total & 20 & 100 & 100 & & \\
\hline \multicolumn{6}{|l|}{ Há quanto tempo perde urina } \\
\hline$<1$ ano & 8 & 40,0 & 40,0 & 40,0 & \multirow{2}{*}{0,061} \\
\hline 1 a 3 anos & 6 & 30,0 & 30,0 & 70,0 & \\
\hline$>4$ anos & 6 & 30,0 & 30,0 & 100 & \\
\hline Total & 20 & 100 & 100 & & \\
\hline \multicolumn{6}{|l|}{ Chega ao banheiro a tempo para urinar } \\
\hline Sempre & 4 & 20,0 & 20,0 & 20,0 & \multirow{5}{*}{0,073} \\
\hline Na maioria das vezes & 7 & 35,0 & 35,0 & 55,0 & \\
\hline Âs vezes & 5 & 25,0 & 25,0 & 80,0 & \\
\hline Nunca & 4 & 20,0 & 20,0 & 100 & \\
\hline Total & 20 & 100 & 100 & & \\
\hline \multicolumn{6}{|c|}{ Acorda à noite quando sente vontade de urinar } \\
\hline Sim, acorda e vai ao banheiro & 18 & 90,0 & 90,0 & 90,0 & \multirow{3}{*}{$0,002 *$} \\
\hline Sim, acorda, mas não vai ao banheiro & 1 & 5,0 & 5,0 & 95,0 & \\
\hline Não acorda & 1 & 5,0 & 5,0 & 100 & \\
\hline Total & 20 & 100 & 100 & & \\
\hline
\end{tabular}


Tabela 2. Continuação.

\begin{tabular}{|c|c|c|c|c|c|}
\hline Variáveis & $\mathrm{n}$ & $\%$ & \% válido & $\%$ acumulado & Valor $p$ \\
\hline \multicolumn{6}{|l|}{ Condição ao perder urina } \\
\hline Somente aos grandes esforços & 6 & 30,0 & 30,0 & 30,0 & \multirow{4}{*}{0,076} \\
\hline Ao tossir, rir, espirrar & 10 & 50,0 & 50,0 & 80,0 & \\
\hline Ao caminhar rápido ou correr & 1 & 5,0 & 5,0 & 85,0 & \\
\hline Com movimentos leves, mesmo deitado & 3 & 15,0 & 15,0 & 100 & \\
\hline Total & 20 & 100 & 100 & & \\
\hline \multicolumn{6}{|l|}{ Perde urina durante a relação sexual } \\
\hline Sim & 2 & 10,0 & 10,0 & 10,0 & \multirow{2}{*}{$0,002^{*}$} \\
\hline Não & 18 & 90,0 & 90,0 & 100 & \\
\hline Total & 20 & 100 & 100 & & \\
\hline \multicolumn{6}{|l|}{ Frequência de perda urinária } \\
\hline Menos de uma vez ao mês & 4 & 20,0 & 20,0 & 20,0 & \multirow{4}{*}{0,761} \\
\hline Uma ou várias vezes por semana & 5 & 25,0 & 25,0 & 45,0 & \\
\hline Todos os dias e algumas noites & 5 & 25,0 & 25,0 & 70,0 & \\
\hline Dia e noite & 5 & 25,0 & 25,0 & 95,0 & \\
\hline Todos os dias & 1 & 5,0 & 5,0 & 100 & \\
\hline Total & 20 & 100 & 100 & & \\
\hline \multicolumn{6}{|l|}{ Quantidade de perda urinária } \\
\hline Sensação de estar levemente úmido & 1 & 5,0 & 5,0 & 5,0 & \multirow{4}{*}{0,75} \\
\hline Sensação de estar úmido & 13 & 65,0 & 65,0 & 70,0 & \\
\hline Sensação de estar molhado & 5 & 25,0 & 25,0 & 95,0 & \\
\hline Sensação de estar encharcado & 1 & 5,0 & 5,0 & 100 & \\
\hline Total & 20 & 100 & 100 & & \\
\hline \multicolumn{6}{|l|}{ Recurso para conter a perda de urina } \\
\hline Não usam & 14 & 70,0 & 70,0 & 70,0 & \multirow{3}{*}{$0,047 *$} \\
\hline Absorvente & 5 & 25,0 & 25,0 & 95,0 & \\
\hline Fralda & 1 & 5,0 & 5,0 & 100 & \\
\hline Total & 20 & 100 & 100 & & \\
\hline \multicolumn{6}{|l|}{ Mudanças em hábitos de vida diários } \\
\hline Não houve & 8 & 40,0 & 40,0 & 40,0 & \multirow{5}{*}{0,231} \\
\hline Sono & 9 & 45,0 & 45,0 & 85,0 & \\
\hline Sono, sair de casa e ingestão hídrica & 1 & 5,0 & 5,0 & 90,0 & \\
\hline Deambulação, sair de casa & 1 & 5,0 & 5,0 & 95,0 & \\
\hline Sono, deambulação & 1 & 5,0 & 5,0 & 100 & \\
\hline Total & 20 & 100 & 100 & & \\
\hline \multicolumn{6}{|c|}{ Incontinência afeta suas relações sociais/familiar } \\
\hline Não & 1 & 5,0 & 5,0 & 5,0 & \multirow{2}{*}{$0,002 *$} \\
\hline $\operatorname{Sim}$ & 19 & 95,0 & 95,0 & 100 & \\
\hline Total & 20 & 100 & 100 & & \\
\hline
\end{tabular}

*significância estatística - teste do $\chi^{2}(p \leq 0,05)$. 
Tabela 3. Análise estatística dos escores obtidos para a Escala de Autoestima Rosenberg/UNIFESP-EPM e para a Escala de Investimento Corporal.

\begin{tabular}{|c|c|c|c|c|c|c|}
\hline \multirow{2}{*}{$\begin{array}{l}\text { Estatística } \\
\text { descritiva }\end{array}$} & \multirow{2}{*}{$\begin{array}{c}\text { Escala de Autoestima } \\
\text { Rosenberg/UNIFESP-EPM } \\
\text { Escore total }\end{array}$} & \multicolumn{4}{|c|}{$\begin{array}{c}\text { Escala de Investimento Corporal } \\
\text { Domínios }\end{array}$} & \multirow{2}{*}{ Valor $\mathrm{p}$} \\
\hline & & $\begin{array}{l}\text { Escore } \\
\text { total }\end{array}$ & $\begin{array}{l}\text { Imagem } \\
\text { corporal }\end{array}$ & $\begin{array}{l}\text { Cuidado } \\
\text { pessoal }\end{array}$ & $\begin{array}{l}\text { Toque } \\
\text { pessoal }\end{array}$ & \\
\hline Média & 24,95 & 82,80 & 17,95 & 35,55 & 18,00 & \\
\hline Mediana & 25,00 & 82,00 & 18,00 & 37,00 & 18,00 & 0,002 \\
\hline Moda & 25 & 73 & 18 & 40 & 18 & \\
\hline Desvio padrão & 1,468 & 7,571 & 1,538 & 4,334 & 1,777 & \\
\hline
\end{tabular}

Teste de Kruskal-Wallis e de Spearman $(p \leq 0,05)$

idade de 64 anos, mulheres, e indivíduos casados, fumantes, e de cor branca. Vários estudos epidemiológicos revelam uma prevalência de incontinência urinária de $26,5 \%$ em mulheres de 35 a 64 anos e $41 \%$ naquelas acima de 65 anos, porém existem algumas pesquisas que descrevem uma taxa de 49,6\% em mulheres acima dos 20 anos e $45 \%$ nas que se encontram entre 30 e 90 anos ${ }^{20-23}$.

A prevalência da incontinência urinária aumenta com a idade; portanto, esta condição se tornará extremamente comum com o envelhecimento da população. Os fatores de risco frequentemente apontados são, dentre outros: idade avançada, elevado índice de massa corporal (IMC), raça branca, paridade, menopausa, histerectomia e comorbidades como depressão e diabetes. A incontinência urinária e o declínio da saúde são comumente vistos como parte natural do envelhecimento. Dessa forma, apesar de ser uma das doenças crônicas mais prevalentes, não é frequentemente reconhecida pelo sistema de cuidado à saúde ${ }^{24-27}$.

Neste estudo, 16 (80\%) participantes tinham ensino fundamental incompleto e sua renda familiar mensal era de até três salários-mínimos (um salário-mínimo $=\mathrm{R} \$ 788,00$ ). Tais achados coincidem com os encontrados por vários autores $^{5,27-31}$, e revelam um perfil preocupante ao reportar à cidadania e ao respeito aos direitos humanos, pois sabemos que quanto mais baixa a escolaridade, mais desfavorável é o capital linguístico dos sujeitos para questionar os profissionais acerca de seus problemas de saúde, do cuidado a ser efetivado e sobre os direitos que lhes são inerentes. Vale ressaltar que tal situação não interfere na atuação junto a essas pessoas, pois a interação entre usuário, serviço e profissionais de saúde tem proporcionado a superação das dificuldades impostas por essa variável ${ }^{32}$.

Com relação às características de perda urinária, 15 (75\%) entrevistados relataram que nunca foram ao médico em função da perda urinária; 8 (40\%) perdem urina há menos de um ano; 10 (50\%) perdem urina ao tossir, rir e espirrar; 13 (65\%) têm sensação de estarem úmidos e 19 (95\%) afirmaram que a incontinência afeta as suas relações sociais e familiar. Essas informações corroboram os resultados de vários estudos $5,21-23,26,27,30$.

Com o aumento da perspectiva de vida das populações em geral, um número crescente de idosos com diabetes está surgindo e, consequentemente, os casos de incontinência urinária vêm aumentando. Muitos deles não serão diagnosticados, em razão de os portadores não buscarem tratamento e despreparo de alguns profissionais de saúde na abordagem, no diagnóstico e no cuidado. Diversos casos não serão descobertos, porque o paciente com incontinência tem medo ou vergonha de relatar à família ou ao próprio profissional de saúde.

Um estudo que avaliou os conhecimentos a respeito das vivências com a perda urinária entre mulheres brasileiras e em que foi utilizada a técnica de análise de conteúdo com abordagens, revelou que as mulheres temem reações restritivas tanto da sociedade como do profissional de saúde. Os significados da perda urinária e os sentimentos vivenciados aumentaram ainda mais as dificuldades de as mulheres expressarem seu problema para esses profissionais. $O$ presente estudo mostra que o significado da incontinência urinária perpassa os problemas econômicos e educacionais da nossa população e evidencia as inquietações psicológicas e sociais vivenciadas por esse grupo de mulheres, ao legitimar que a incontinência urinária deve ser escondida como uma forma de proteção aos sentimentos de angústia, medo e vergonha, e pode levar a uma vida solitária como alternativa de sobrevivência diante das marcas profundas de um estigma que interfere na sua qualidade de vida ${ }^{33}$.

A literatura ressalta que a intensidade com que ocorre a incontinência urinária (pequena, moderada ou severa) vai influenciar a qualidade de vida, autoestima, autoimagem 
do paciente incontinente, ou seja, quanto maior o volume urinário perdido, maior será a implicação negativa na vida desses indivíduos ${ }^{34}$. A pessoa com incontinência urinária sofre impactos físico, psicológico e emocional, bem como uma súbita destruição de sua imagem corporal e da autoestima, impactando de forma negativa no estabelecimento das ordens social, doméstica e sexual. Além disso, pode exacerbar o medo, a dor e o sofrimento, sentindo rejeição por si próprio e pelos semelhantes, fator importante perante os amigos e familiares, bem como ter alterações em outras dimensões, tais como o humor, que tem como consequência o isolamento da sociedade e família e privação de lazer.

O indivíduo com incontinência urinária, ao ter as estruturas anatômica e fisiológica modificadas, defronta-se com seus conflitos e fantasias em função da imagem que ele faz do corpo, como também daquela que fazem dele as pessoas que o cercam. Este fato favorece a perda da autoestima, uma vez que, ao lidar com a incontinência urinária, esse indivíduo atrai a atenção das pessoas aos valores relacionados à eliminação de urina e odor e o que antes era naturalizado, passa a ser alvo de reflexão e revisão. $\mathrm{O}$ isolamento social é algo visível, no entanto, é importante destacar que diante dessa realidade é imperativo que haja interação social, já que esse processo contribuirá para o restabelecimento da percepção da pessoa relacionada ao seu corpo e a sua própria autoimagem e, principalmente, contribuirá para a superação da solidão ${ }^{35,36}$.

No presente estudo, a autoimagem foi avaliada utilizando-se a versão brasileira da Escala de Investimento Corporal, em que o escore médio no domínio imagem corporal foi de 17,97 e no toque corporal foi de 18,00, os quais são considerados baixos. Isso mostra o quanto o portador de incontinência urinária está insatisfeito com a sua imagem corporal, motivo pelo qual muitas vezes eles recusam-se a ser tocados. Quanto ao domínio cuidado pessoal, o escore médio foi de 35,55. Ao adquirirem a incontinência urinária, os indivíduos sentem que seus corpos foram modificados, pois estão eliminando urina espontaneamente e exalando odor, mas assim mesmo se cuidam e tentam melhorar a sua aparência, tomando banho toda vez que percebem que escapou urina, trocando o absorvente, a roupa íntima, o que contribui para evitar o cheiro desagradável de urina, que tanto incomoda a quem tem essa alteração.

Para avaliar a autoestima, utilizou-se a versão brasileira da Escala de Autoestima Rosenberg/UNIFESP-EPM e o escore total médio foi de 24,95 . Tendo em vista que o valor zero significa melhor autoestima e 30, a pior, pode-se dizer que aquela dos portadores de incontinência urinaria é baixa.

O nosso corpo é referência para a interpretação das manifestações corporais. Este, a matéria de vinculação das pessoas ao mundo, antes de ser um objeto de intervenção das ciências biomédicas, é a dimensão do nosso próprio ser. O corpo é o local da experiência e da prática da vida e, portanto, a reflexão sobre este é posterior à sua experiência. Nesse enfoque, o modo como as pessoas vivenciam uma doença é primeiramente uma síntese entre corpo e cultura para, depois, ser uma reflexão sobre a doença ${ }^{37}$.

A imagem corporal e a autoestima, na contemporaneidade, estão relacionadas à juventude, beleza, vigor, integridade e saúde, e aqueles que não correspondem a esse conceito de padrão corporal poderão experimentar um significativo senso de rejeição ${ }^{26}$.

Nas alterações psíquicas, a mudança da autoimagem e autoestima é um aspecto que se sobressai, uma vez que o paciente terá de redefinir sua imagem corporal, sua maneira de se vestir e interagir com seu corpo. Quanto ao aspecto social, a presença da incontinência urinária repercute tanto no nível familiar, quanto no lazer e nas atividades laborais do paciente. As alterações nas atividades de lazer relacionam-se quase sempre à vergonha, fragilidade, impotência ou presença aparente de odor e umidade ${ }^{38,39}$.

Este estudo reforça a necessidade de se redirecionar a atenção à saúde dos pacientes com incontinência urinária, buscando identificar, no cotidiano dos serviços de saúde, hospitais ou ambulatórios, e em Programas de Saúde da Família, a presença de alterações da autoestima, autoimagem e da própria sexualidade. Devem ser atendidas as principais necessidades de cuidado aos pacientes com incontinência urinária em seu cotidiano e é fundamental o conhecimento do cuidador para lidar com as incapacidades dessa população.

Enfim, os serviços de saúde ainda precisam se preparar melhor para atender o usuário que tem incontinência urinária. Faltam profissionais treinados para acolher esse tipo de paciente. Precisamos cuidar do corpo e, principalmente, do psicológico desse tipo de usuário. $\mathrm{O}$ diálogo, o acolhimento e o apoio vão ajudá-lo a não se excluir por vergonha do próprio corpo perante a sociedade.

Diante das necessidades surgidas nas últimas décadas, com o aumento do número de pacientes com doenças crônicas que podem levar o indivíduo a conviver com incontinência urinária, torna-se imprescindível redirecionar a formação acadêmica e a qualificação dos profissionais de 
saúde, valorizando não somente o conteúdo, mas também a prática assistencial prestada, sendo essencial que esses profissionais se tornem conscientes da importância de o paciente realizar autocuidado. Estudos futuros devem ser conduzidos, visando à ampliação do tamanho da amostra e à compreensão da magnitude de vida desses sujeitos. Este estudo apresentou, como limitação, o número de participantes, sendo necessária a realização de mais pesquisas na área para maior aprofundamento no tema.

\section{CONCLUSÃO}

Os pacientes deste estudo com incontinência urinária tiveram alterações no escore da Escala de Autoestima Rosenberg/
UNIFESP-EPM e da Escala de Investimento Corporal nos domínios 'imagem corporal' e 'toque corporal', mostrando sentimentos negativos em relação ao próprio corpo.

\section{AGRADECIMENTOS}

Os autores TFO e WAP agradecem pela bolsa de estudos do Programa de Bolsa de Iniciação Científica (PROBIC) da Fundação de Amparo à Pesquisa do Estado de Minas Gerais (FAPEMIG). Esta instituição pública independente não teve participação no desenho e na conduta deste estudo, incluindo coleta de dados, gerenciamento, análise e interpretação de dados, preparo, revisão e aprovação do manuscrito.

\section{REFERÊNCIAS}

1. Feldner PCJr, Bezerra LR, Girão MJ, Sartori MG, BacaratEC, Lima GR. A importância do diagnóstico da deficiência esfincteriana uretral intrínseca em mulheres com incontinência urinária de esforço. Femina. 2003;31(10):851-4.

2. Miner PB Jr. Economic and personal impact of fecal and urinary incontinence. Gastroenterology. 2004;126(1 Suppl 1):S8-13.

3. Slieker-ten Hove MC, Pool-Goudzward AL, Eijkemans MJ, Steegers-Theunissen RP, Burger CW, Vierhout ME. Prevalence of double incontinence, risks and influence on quality of life in a general female population. Neurourol Urodyn. 2010;29(4):545-50.

4. Dolan LM, Walsh D, Hamilton S, Marshall K, Thompson K, Ashe RG. A study of quality of life in primigravidae with urinary incontinence. Int Urogynecol J Pelvic Floor Dysfunct. 2004;15(3):160-4.

5. Santos CR, Santos VL. Prevalence of self-reported double incontinence in the urban population of a Brazilian city. Neurourol Urodyn. 2011;30(8):1473-9.

6. Troncon LE, Lopes RP, Simão MN, Iquegami M, Rosa-e-Silva L, Nobre-e-Souza MA, et al. Frequency of digestive symptoms in Brazilian patients with Diabetes Mellitus. Rev Ass Med Bras. 2001;47(2):157-64.

7. Tavares DM, Rodrigues FR, Silva CG, Castro Miranzi SS. Characterization of elderly diabetics receiving secondary care. Cienc Saúde Colet. 2007;12(5):1341-52.

8. Izci Y, Topsever P, Filiz TM, Çınar ND, Uludağ C, Lagro-Janssen $T$. The association between diabetes mellitus and urinary incontinence in adult women. Int Urogynecol J Pelvic Floor Dysfunct. 2009;20(8):947-52.

9. Jesus Menezes MA, Hashimoto SY, Gouveia Santos VL. Prevalence of urinary incontinence in a community sample from the city of São Paulo. J Wound Ostomy Continence Nurs. 2009;36(4):436-40.
10. Cássia Domansky R, Gouveia Santos VL. Cross-cultural adaptation and validation of the Portuguese version of the Bowel Function in the Community instrument. J Wound Ostomy Continence Nurs. 2007;34(6):671-7.

11. Dini GM, Quaresma MR, Ferreira LM. Adaptação cultural e validação da versão brasileira da escala de auto-estima de Rosenberg. Rev Soc Bras Cir Plast. 2004;19(1):41-52.

12. Bicalho MB, Lopes $\mathrm{MH}$. The impact of urinary incontinence of the lives of wives of men with incontinence: an integrative review. Rev Esc Enferm USP. 2012;46(4):1009-14.

13. Emmons KR, Robinson JP. Urinary incontinence severity and quality-of-life instruments. J Wound Ostomy Continence Nurs. 2013;40(4):350-4.

14. Lombraña M, Izquierdo L, Gómez A, Alcaraz A. Impact of a nurse-run clinic on prevalence of urinary incontinence and everyday life in men undergoing radical prostatectomy. J Wound Ostomy Continence Nurs. 2013;40(3):309-12.

15. Lobchuk MM, Rosenberg F. A qualitative analysis of individual and family caregiver responses to the impact of urinary incontinence on quality of life. J Wound Ostomy Continence Nurs. 2014;41(6):589-96.

16. Moccellin AS, Rett MT, Driusso P. Urinary incontinence during pregnancy: the effects on quality of life. Rev Bras Saude Matern Infant. 2014;14(2):147-54.

17. Pereira MT, Salomé GM, Openheimer DG, Espósito VH, de Almeida SA, Ferreira LM. Feelings of powerlessness in patients with diabetic foot ulcers. Wounds. 2014;26(6):172-7.

18. de Almeida SA, Salomé GM, Dutra RA, Ferreira LM. Feelings of powerlessness in individuals with either venous or diabetic foot ulcers. J Tissue Viability. 2014;23(3):109-14.

19. Salomé GM, Blanes L, Ferreira LM. Functional capability of patients with diabetes with foot ulceration. Acta Paul Enferm. 2009;22(4):412-6. 
20. Santos CR, Santos VL. Epidemiology of combined urinary and fecal incontinence. Acta Paul Enferm. 2009;22(3):328-30.

21. Lopes DB, Praça NS. Prevalence and related factors of selfreported urinary incontinence in the postpartum period. Acta Paul Enferm. 2012;25(4):574-80.

22. Silva AP, Santos VL. Prevalence of urinary incontinence in hospitalized patients Rev Esc Enferm USP. 2005;39(1):36-45.

23. Souza Santos CR, Santos VL. Prevalence of urinary incontinence in a random sample of the urban population of Pouso Alegre, Minas Gerais, Brazil. Rev Latino-Am Enferm. 2010;18(5):903-10.

24. Fenner DE, Trowbridge ER, Patel DA, Fultz NH, Miller JM, Howard D, et al. Establishing the prevalence of incontinence study: racial differences in women's patterns of urinary incontinence. J Urol. 2008;179(4):1455-60.

25. Goode PS, Burgio KL, Redden DT, Markland A, Richter HE, Sawyer $P$, et al. Population based study of incidence and predictors of urinary incontinence in black and white older adults. J Urol. 2008;179(4):1449-53.

26. Meirelles CA, Ferraz CA. Evaluation of the process of intestinal stoma demarcation and late complications in ostomized patients. Rev Latino-Am Enferm. 2001;9(5):32-8.

27. Danforth KN, Townsend MK, Lifford K, Curhan GC, Resnick NM, Grodstein F. Risk factors for urinary incontinence among middleaged women. Am J Obstet Gynecol. 2006;194(2):339-45.

28. Melville JL, Katon W, Delaney K, Newton K. Urinary incontinence in US women: a population-based study. Arch Intern Med. 2005;165(5):537-42.

29. Lopes MH, Higa R. Urinary incontinence restrictions in women's life. Rev Esc Enferm USP. 2006;40(1):34-41.

30. Barbosa AM, Marini G, Piculo F, Rudge CV, Calderon IM, Rudge MV. Prevalence of urinary incontinence and pelvic floor muscle dysfunction in primiparae two years after cesarean section: cross-sectional study. São Paulo Med J. 2013;131(2):95-9.
31. Amaral SS, Teixeira MG, Brito SL, Amaral GA, Jorge JM, HabrGama A, et al. Prevalence of fecal incontinence in the diabetic an epidemiological study in those diabetics attended as outpatients at the Hospital das Clinicas da Faculdade de Medicina da Universidade de Sao Paulo. Rev Hosp Clin Fac Med Univ São Paulo. 1997;52(6):295-301.

32. Brasil. Ministério da Saúde. Secretaria de Atenção à Saúde. Portaria $n^{\circ}$ 400, de 16 de novembro de 2009. Brasília (DF): Ministério da Saúde; 2009.

33. Higa R, Rivorêdo CR, Campos LK, Lopes MH, Turato ER. Life experiences of Brazilian women with urinary incontinence. Texto Contexto Enferm. 2010;19(4):627-35.

34. Butler RN, Maby JI, Montella JM, Young GP. Urinary incontinence: keys to diagnosis of the older woman. Geriatrics. 1999;54(10):29-30.

35. Gentile S, Bronzino P, Persico M, Marmo R, Costume A, Contaldi $P$, et al. Diabetic neuropathy. III: Autonomic neuropathy. Genito-urinary system. Minerva Med. 1984;75(17):967-74

36. Souza PC, Costa VR, Maruyama SA, Costa AL, Rodrigues $A E$, Navarro JP. As repercussões de viver com uma colostomia temporária nos corpos: individual, social e político. Rev Eletr Enferm. 2011;13(1):50-9.

37. Santos VL, Sawaia BB. A bolsa na mediação "estar ostomizado" - "estar profissional": análise de uma estratégia pedagógica. Rev Latino-Am Enferm. 2000;8(3):40-50.

38. Costa VF, Alves SG, Eufrásio C, Salomé GM, Ferreira LM. Assessing the body image and subjective wellbeing of ostomists living in Brazil. Gastrointestinal Nurs. 2014;12(5):37-47.

39. Alves SG, Reis BC, Gardona RG, Vilela LH, Salomé GM. Associação dos fatores sociodemográficos e da lesão relacionados ao sentimento de impotência e esperança em indivíduos com úlcera venosa. Rev Bras Cir Plast. 2014;28(4):672-80. 